\title{
Online-Only Supplemental Material \\ Changes in Statin Utilization among US Adults with Diabetes: A Population-Based Analysis of NHANES 2011-2018
}

\section{Supplement Appendix.}

Study Participants and Design:

NHANES offers a representative sample of the noninstitutionalized civilian US population using a stratified, multistage probability sample. Data were collected through interviews and physical examination; specific protocols can be found the on the NHANES website

(https://www.cdc.gov/nchs/nhanes/index.htm). The analysis included adults 40-75 years old with diabetes participating in the mobile examination component without end-stage kidney disease or evidence of pregnancy. Recent survey cycles were pooled into 2 cohorts, covering 4 years prior (2011-2014) and 4 years after (2015-2018) the 2015 statement. Given the pre-specified timing of the cohorts, no washout period was used. The Standards of Medical Care have been published in January each year since 2002; it was assumed providers would anticipate changes early in the cycle.

Diabetes was defined as a self-reported diagnosis of diabetes or $\mathrm{HbA} 1 \mathrm{c} \geq 6.5 \%$. Statin use was ascertained via interview and medication container review. The 2008-2014 Standards of Medical Care were used to determine statin indication before 2015. Cardiovascular risk factors included hypertension (self-reported or systolic blood pressure $>140 \mathrm{mmHg}$ ), smoking (current smoker plus exposure $>100$ cigarettes), albuminuria (albumin/creatinine ratio $>30 \mathrm{mg} / \mathrm{g}$ ), and close biological relative with a history of myocardial infarction. Overt ASCVD included a composite of self-reported coronary artery disease, angina, myocardial infarction, and stroke. Hyperlipidemia was not considered a risk factor due to insufficient data on the pre-statin lipid profile. History of hyperlipidemia included self-report or total cholesterol >200 mg/dl.

The primary outcome was the change in statin utilization among participants with diabetes but without ASCVD risk factors (no ASCVD group) controlling for the change among those with 
ASCVD or ASCVD risk factors (control group). It was hypothesized that a greater increase in statin therapy would be seen among people without ASCVD risk factors, as the 2015 recommendations expanded statin utilization to include this population.

\section{$\underline{\text { Data Analysis }}$}

Demographic data were presented as proportions (\%) or means with standard errors (SE). The percentage of adults taking statins was calculated and compared across study periods. The primary analysis was conducted using the quasi-experimental difference-in-differences technique to model the effect of the 2015 ADA position statement on statin utilization among patients with diabetes without ASCVD risk factors. The difference-in-differences estimates the relative change in statin utilization over time. With difference-in-difference modeling, changes in the outcome over time from the control group are subtracted from those of the no ASCVD group. This approach reduces bias in the post-recommendation period by accounting for changes in statin utilization over time due to other causes by comparing the no ASCVD group to participants who previously met clinical practice recommendations for statin therapy (and would therefore not be expected to have increased utilization in response to the 2015 recommendations). As a result, this design is well suited for evaluating guideline changes by isolating the effects where the new statin therapy recommendations apply (i.e. the no ASCVD group) relative to the control group and pre-existing trends. This technique was operationalized in a logistic regression model with an interaction term between the dichotomous variables: study period (where 2011-2014= 0 and 2015-2018=1) and group (where control group=0 and no ASCVD group $=1)$. The resulting model was $\log -$ odds $($ statin use $)=\beta_{0}+\beta_{1}($ study period $)+$ $\beta_{2}($ group $)+\beta_{3}($ study period $*$ group $)$. The validity of the underlying parallel trend assumption was assessed by visual inspection of the trend for two pre-recommendation observations. Risk factors for not receiving a recommended statin were identified by the inclusion of clinical and 
socioeconomic covariates in an adjusted logistic regression model. For highly correlated covariates, only one was entered into the regression analysis.

Analyses were weighted to represent the US population and conducted using the Analysis of Complex Survey Samples, 'survey', package in RStudio 3.6.1 (R Foundation for Statistical Computing, Vienna, Austria) to account for the complex survey design. The sample weights were constructed based on the assigned MEC sample weight, the measure of the number of people in the population represented by each sampled person participating in the exam survey component, and the number of survey cycles included in each cohort. $P$ values $<0.05$ were considered statistically significant. 
Supplementary Table S1. Baseline demographics and clinical characteristics

\begin{tabular}{|c|c|c|c|c|}
\hline & \multicolumn{2}{|c|}{$\begin{array}{l}\text { ASCVD/ASCVD Risk Factors } \\
\text { (control group) }\end{array}$} & \multicolumn{2}{|c|}{$\begin{array}{l}\text { No ASCVD Risk Factors } \\
\text { (No ASCVD group) }\end{array}$} \\
\hline & Pre & Post & Pre & Post \\
\hline Sample N & 1099 & 1276 & 195 & 236 \\
\hline Weighted N (millions) & 17.6 & 20.2 & 2.9 & 3.7 \\
\hline Statin Utilization (\%) & 51.1 & 54.8 & 32.7 & 43.0 \\
\hline Age (years) & $59.1(0.4)$ & $60.0(0.5)$ & $54.8(0.8)$ & $56.7(1.0)$ \\
\hline Male & 51.8 & 53.8 & 50.7 & 58.1 \\
\hline $\begin{array}{r}\text { White } \\
\text { Black } \\
\text { Mexican American } \\
\text { Hispanic } \\
\text { Asian } \\
\text { Other }\end{array}$ & $\begin{array}{l}59.3 \\
16.8 \\
9.2 \\
6.2 \\
4.9 \\
3.5\end{array}$ & $\begin{array}{l}57.1 \\
15.2 \\
9.0 \\
6.9 \\
6.0 \\
5.7\end{array}$ & $\begin{array}{l}45.4 \\
12.8 \\
15.3 \\
8.0 \\
13.0 \\
5.5\end{array}$ & $\begin{array}{l}50.2 \\
8.6 \\
18.3 \\
8.0 \\
10.5 \\
4.3\end{array}$ \\
\hline $\begin{array}{r}\text { Education } \\
\text { Less than high school } \\
\text { High school } \\
\text { Some college } \\
\text { College graduate }\end{array}$ & $\begin{array}{l}23.4 \\
25.9 \\
33.3 \\
17.4 \\
\end{array}$ & $\begin{array}{l}18.2 \\
25.9 \\
34.5 \\
21.4\end{array}$ & $\begin{array}{l}21.2 \\
17.6 \\
27.6 \\
33.6\end{array}$ & $\begin{array}{l}19.8 \\
15.9 \\
23.3 \\
41.0\end{array}$ \\
\hline $\begin{array}{r}<\$ 20,000 \\
\$ 20,000-\$ 45,000 \\
\$ 45,000-\$ 75,000 \\
>\$ 75,000\end{array}$ & $\begin{array}{l}21.7 \\
31.3 \\
22.3 \\
24.6\end{array}$ & $\begin{array}{l}16.3 \\
32.0 \\
19.7 \\
32.0\end{array}$ & $\begin{array}{l}19.2 \\
37.5 \\
19.9 \\
23.3\end{array}$ & $\begin{array}{l}9.2 \\
27.9 \\
20.1 \\
42.8\end{array}$ \\
\hline No health insurance & 13.4 & 8.3 & 17.9 & 9.2 \\
\hline No routine place for care & 5.5 & 6.9 & 8.7 & 9.0 \\
\hline No routine care in last year & 7.4 & 5.0 & 9.0 & 7.8 \\
\hline $\mathrm{HbA}_{1 \mathrm{c}}(\%)[\mathrm{mmol} / \mathrm{mol}]$ & $7.6[60](0.1)$ & $7.4[57](0.1)$ & $7.4[57](0.2)$ & $7.3[56](0.1)$ \\
\hline $\mathrm{SBP}(\mathrm{mmHg})$ & $120.7(0.9)$ & $132.1(0.7)$ & $119.3(1.2)$ & $120.0(1.2)$ \\
\hline Hyperlipidemia (\%) & 76.2 & 75.0 & 69.7 & 69.4 \\
\hline Total cholesterol (mg/dL) & $184.9(2.4)$ & $183.1(2.3)$ & $189.0(3.4)$ & $184.8(5.0)$ \\
\hline
\end{tabular}

Abbreviations: ASCVD=Atherosclerotic Cardiovascular Disease Data are represented as percent or mean (standard error). Weighted $\mathrm{N}$ was calculated using mobile examination survey weights divided by the number of survey cycles to represent the US population in each cohort. Pre indicates from 2011 to 2014, and Post indicates from 2015 to 2018 